Apuntes Universitarios, 2020: 10(2), Abril-Junio

ISSN: 2304-0335 DOI: https://doi.org/10.17162/au.v10i2.434

\title{
Las TIC y su influencia en el desarrollo psicosocial
}

\section{ICT and its influence on psychosocial development}

\author{
Kennya Guzmán Huayamave ${ }^{1}$, Patricia Arriaga Hachi² y Alison Cobos Díaz \\ Universidad Laica Vicente Rocafuerte de Guayaquil, Ecuador ${ }^{123}$
}

iD Orcid ID: https://orcid.org/0000-0003-1204-27291

iD Orcid ID: https://orcid.org/0000-0001-6890-1778²

iD Orcid ID: https://orcid.org/0000-0002-1343-14113

Recibido: 01 de julio 2019

Aceptado: 15 de diciembre 2019

\section{Resumen}

El presente artículo describe el valor de las Tecnologías de la Información y la Comunicación (TIC) como recurso didáctico que abre oportunidades para el desarrollo del conocimiento. Al estar inmersos en una sociedad que los niños son nativos digitales, el docente requiere incorporar tecnología en sus procesos educativos a fin de potenciar el desarrollo psicosocial de sus alumnos. En este contexto, la investigación tiene como objetivo promover espacios de reflexión para que los escenarios pedagógicos sean cada vez más interactivos con el conocimiento y la socialización de los pares en la escuela "Camino al Espacio" de la ciudad de Guayaquil. Desde esta perspectiva, se utilizaron técnicas cualitativas-cuantitativas con un carácter descriptivo y exploratorio. La metodología considerada fue: observación, entrevista y encuesta. Como conclusión, el desempeño docente debe asumir en su formación el tema de las TIC y en este abordaje, repensar en las experiencias que se construyen en el proceso de enseñanza.

Palabras claves: TIC, aprendizaje, educación y desarrollo

\begin{abstract}
${ }^{1}$ Correspondencia al autor

E-mail: kguzmanh@ulvr.edu.ec
\end{abstract}

This article describes the value of Information and Communication Technologies (ICT) as a teaching resource that opens up opportunities for knowledge development. Being immersed in a society that children are digital natives, the teacher needs to incorporate technology in 
their educational processes in order to enhance the psychosocial development of their students. In this context, the research aims to promote spaces for reflection so that pedagogical scenarios are increasingly interactive with the knowledge and socialization of peers in the "Camino al Espacio" school in the city of Guayaquil. From this perspective, qualitative-quantitative techniques with a descriptive and exploratory character were used. The methodology considered was: observation, interview and survey. In conclusion, the teaching performance must assume in its formation the topic of ICT and in this approach, rethink the experiences that are built in the teaching process.

Keywords: ICT, learning, education and development

\section{Introducción}

Uno de los principales desafíos que genera debate a nivel académico, y se ha intensificado en los últimos años por su gran valor en la comunicación es la presencia de las TIC en los ambientes de aprendizaje y educación. Al respecto, UNESCO (2013) considera que las TIC: "constituyen sobre todo nuevas conversaciones, estéticas, narrativas, vínculos relacionales, modalidades de construir identidades y perspectivas sobre el mundo" (p.16). En este caso, las exigencias del siglo XXI conducen al manejo de las tecnologías y en esta modalidad cultural, el docente cumple un papel fundamental como gestor del aprendizaje.

Cabe resaltar que, en el estado mundial de la infancia UNICEF (2017) refiere que: "La tecnología digital ya ha cambiado el mundo y, a medida que aumenta el número de niños que se conectan en línea en todos los países, está cambiando cada vez más su infancia” (p.7). De acuerdo a lo expresado, en Ecuador preocupa la presencia de ambientes educativos de aprendizaje tecnológicos que no integran la teoría con la práctica; y esto-en su organización áulica_-no propicia posibilidades de desarrollo.

De lo expuesto, las TIC pueden convertirse en entornos dinámicos y colaborativos de aprendizaje, que favorecen el panorama digital con sus múltiples beneficios. Al hilo de lo señalado, Espinoza y Rodríguez (2017) consideran que:

Las nuevas tecnologías al alcance de los niños y jóvenes desde su nacimiento, ofrecen no solo nuevas posibilidades de acceso a la información y comunicación, sino también nuevos paradigmas de relación con el contexto social, a través de la modificación y generación de nuevas habilidades socio afectivo. Es importante conocer de qué manera las formas de socialización se han transformado a partir del uso de la tecnología. (p. 5) 
En consonancia con los autores, la revolución digital tiene también una connotación psicológica y social, ya que las relaciones interpersonales traspasan fronteras, edades y toda clase social; así como el impacto que se genera en la vida de quienes viven conectados. Ante esto, Fernández (2016) sostiene que: "también padres y madres deben aprender a utilizarlas [las TIC] adecuadamente y crear en sus hijos e hijas unos buenos hábitos de uso" (p.4).

A la luz de lo expuesto, Bustamante (2015) considera que la dinámica del aula y los recursos tecnológicos que se utilicen en la educación inicial como parte del Sistema Educativo Nacional deben orientarse con:

teorías constructivistas, con enfoque humanista, donde se integra el desarrollo personal, social, la relación con el medio cultural y natural, así como la comprensión y expresión mediante diferentes lenguajes como parte de un proceso permanente, progresivo e integrado de interrelación con el entorno. (p.16)

A partir de los criterios establecidos, el presente artículo analiza los siguientes aspectos: a). Factor motivacional de la comunidad educativa para generar conocimientos digitales, planificación innovadora, capacitaciones para responder a los desafíos de las tecnologías en los espacios pedagógicos y b). Articular la tecnología y el desarrollo psicosocial de los niños. En consecuencia, la finalidad del presente artículo concibe espacios que intercambian experiencias sobre su práctica educativa a través de la promoción de las TIC.

\section{Aspectos conceptuales}

\section{¿Cuáles son las ventajas de las TIC en los espacios áulicos?}

El considerar las TIC en los niños es un tema que ha generado debates a nivel mundial, debido a la creciente influencia que ejerce la tecnología digital en los procesos áulicos desde temprana edad. En este sentido, Alpízar (2018) sostiene que el uso de las tecnologías en la etapa pre-escolar debe ser considerada como posibilidad para optimizar el quehacer educativo.

Por su parte, Roca ( 2015) recuerda que: "Nuestros niños han nacido en la era digital, pero eso no significa que sepan hacer un buen uso de lo digital” (p. 6). Por ello, la actuación de padres y educadores debe alinearse para favorecer un ambiente saludable; debe llevar a los menores a aprovechar las posibilidades de las TIC y reducir sus riesgos. 
En correspondencia con este principio, Briceño, Flores y Gómez (2019) refieren que el uso de las TIC en la primera infancia "es un elemento motivador de los intereses y participación de los estudiantes, ya que pueden avanzar por sus propias capacidades e interactuar con otros para colaborar y aprender usando las tecnologías como mediadoras" (p.22). Por tal motivo, el rol del docente es un pilar fundamental para trabajar desde la alfabetización inicial y reflexionar sobre los diferentes caminos para orientar el uso de las tecnologías.

Es conveniente mencionar que CERLALC (2019) considera la necesidad de implementar investigaciones sobre el uso de las tecnologías en la primera infancia y sostienen en uno de sus apartados que:

Si es cierto que nuestros hijos vivirán en un entorno de cambios continuos, hemos de trasmitirles criterios y certezas, hemos de darles una formación humanística desde la cual podrán abordar serena y críticamente esos cambios, así como contextualizar la información que reciben mediante esas plataformas. (p.19)

En virtud de lo mencionado, la intencionalidad pedagógica del docente en la investigación, genera la siguiente pregunta: ¿De qué manera las prácticas digitales pueden favorecer el desarrollo psicosocial en los niños? En consonancia con lo anterior, se puede afirmar que las TIC facilitan actitudes sociales y suman elementos que resaltan en el siguiente esquema:

Figura 1. Las TIC y la comunicación en el aula
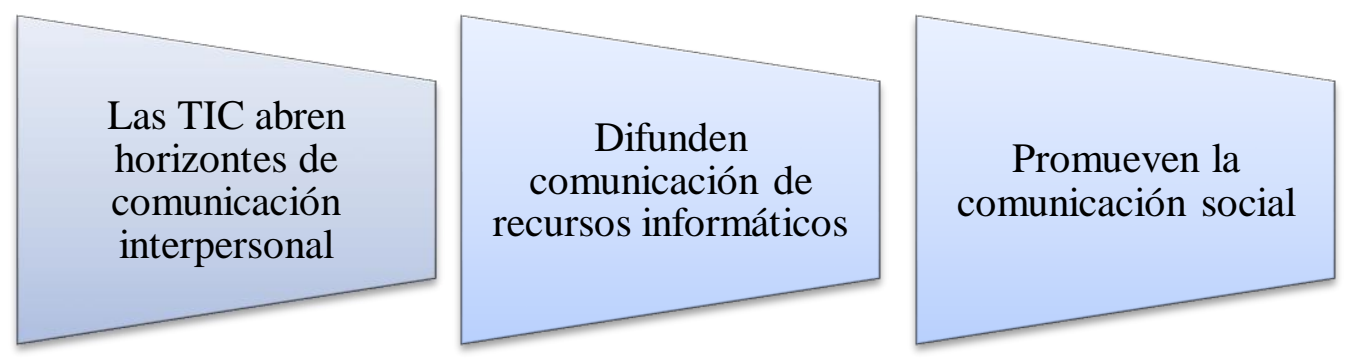

Fuente: Elaboración de las autoras

Al respecto, una mirada interdisciplinar permite contextualizar los procesos cognitivos y sociales con una postura enriquecedora a fin de responder a las necesidades de cada estudiante. Para ello, la planificación curricular, desde la etapa inicial, debe ser 
considerada desde las aristas de las TIC con los paradigmas actuales y en concordancia con las diferentes disciplinas.

Lo expresado reafirma la idea que los niños deben contar con las herramientas informáticas necesarias para optimizar su desarrollo del niño y con ello reducir los riesgos que se presentan en el manejo de la tecnología digital. Por consiguiente, las TIC pueden sensibilizar y aumentar el nivel de comprensión del mundo para los niños y generar en ellos mayor motivación en las propuestas educativas.

En correspondencia con lo expuesto, los nuevos retos de comunicación proporcionan en el contexto educativo los siguientes beneficios:

$>$ Fomenta la creatividad de sus participantes.

> Despierta el espíritu de la investigación ante las nuevas temáticas planteadas.

$>$ Promueve el desarrollo de competencias.

$>$ Respeto por el ritmo de aprendizaje de los estudiantes.

$>$ Estimula el trabajo colaborativo.

Motiva la confianza y el interés por aprender.

Al hilo de lo señalado, conviene enfatizar el papel que el docente de educación infantil tiene en la responsabilidad de asentar bases en el desarrollo humano y cumplir con la optimización de los ambientes de aprendizaje al utilizar los ordenadores, medios visuales, pizarras interactivas, medios sonoros, y todo material en esta línea; además de promover que los niños trabajen de forma individual o grupal.

\section{El uso de las TIC y los comportamientos psicosociales del niño}

En virtud de lo mencionado, las TIC están presente en la cultura actual y ellas inciden en todo lo que realiza el niño; por ello, las tales provocan una fascinación y un flujo de múltiples estímulos para construir su propia realidad y encontrarse ligados al placer que ello proporciona.

Precisamente, pensar en el funcionamiento psicológico del niño o niña requiere estímulos para desarrollar en ellos capacidades físicas, cognitivas, emocionales y sociales; los niños aprenden a través de la acción y exploración del medio que los rodea, en un intercambio activo. Ledesma y Cobos (2018) señalan que los procesos de aprendizaje: 
permanecen sin alteración en su forma estructural, más bien lo que ha cambiado es el acceso que los estudiantes tienen a la información, permitiendo su autonomía en el aprendizaje de acuerdo a su propio ritmo, y facilitando una comunicación directa con el docente mediante el uso de herramientas virtuales. (p.43)

En este orden de ideas, se destaca que las TIC pueden tener una gran injerencia en el desenvolvimiento social del niño; y cuando el docente de inicial lo hace parte de su currículo, los procesos de enseñanza-aprendizaje constituyen una fuente de aprendizaje divertida que modifica positivamente su comportamiento.

En este aspecto, Lemus (2018) refiere que es muy importante considerar las conexiones que se establecen con el medio para el desarrollo psicosocial. De aqui la necesidad de observar los tipos de apego, la relación de los padres, los amigos, diversas perspectivas y aprendizajes que ofrece el contexto.

A partir de los criterios establecidos, las TIC cobran mucha importancia en el desarrollo psicosocial del niño, y Roca (2015) considera que: "Las tecnologías hacen que el aprendizaje se produzca de forma más activa, el niño es el centro" (p.11). Se promueven habilidades cuando las TIC se gestionan en las actividades educativas y motivan al niño para construir el conocimiento.

\section{Tabla 1}

Gestión del docente de educación inicial para el manejo de las TIC en el quehacer educativo de la Escuela Camino al Espacio de la ciudad de Guayaquil, 2018

\begin{tabular}{ll}
\hline Presencia: & Docente motivado con el trabajo de las TIC \\
Conocer & Búsqueda de un aprendizaje digital \\
Circuito de información & Consumo tecnológico \\
Estrategias y acciones & Vincular e hipervincular las actividades pedagógicas
\end{tabular}

Fuente: Elaboración de las autoras con los datos obtenidos de la investigación. 


\section{Materiales y métodos}

\section{Participantes}

Para la presente investigación, se consideró a 8 docentes y 75 niños de 2 a 5 años del nivel inicial 1 y 2 que pertenecen a la Unidad Educativa "Camino al Espacio" de la ciudad de Guayaquil.

\section{Tipo y diseño de la Investigación}

La investigación tiene un enfoque mixto de alcance descriptivo y exploratorio.

\section{Instrumentos}

1. Para los 75 niños del nivel, se trabajó con un registro de observación en el período de cuatro semanas de forma consecutiva, para valorar de forma cualitativa la habilidad social que presentan los niños en un ambiente de aprendizaje sin tecnología.

2. Conviene subrayar que, para evaluar a los 8 docentes que corresponden al sub--nivel 1 y 2 de Educación Inicial, se consideró una encuesta y finalmente se aplicó una entrevista al director.

\section{Análisis de datos}

En el presente estudio se han revisado las variables y los comportamientos que se originan cuando el docente desarrolla su proceso enseñanza-aprendizaje con una didáctica tradicional.

\section{Resultados obtenidos de la observación a los niños del nivel inicial}

Los resultados obtenidos evidencian que los niños siguen la rutina que la maestra presenta en la planificación y recoge en la tabla 2 la frecuencia que se evidencia en los participantes. 


\section{Tabla 2}

Niveles de frecuencia por habilidad social en ambientes de aprendizaje sin tecnología de los niños de Educación Inicial de la Escuela "Camino al Saber" de la ciudad de Guayaquil, 2018

\begin{tabular}{lccc}
\hline Habilidad Social & $\begin{array}{c}\text { Muy } \\
\text { Satisfactorio }\end{array}$ & Satisfactorio & $\begin{array}{c}\text { Poco } \\
\text { Satisfactorio }\end{array}$ \\
\hline Trabajo colaborativo & 5 & 25 & 40 \\
Participación creativa & 2 & 30 & 43 \\
Comportamientos interactivos & 6 & 22 & 47 \\
Manejo de tolerancia & 4 & 33 & 38 \\
\hline
\end{tabular}

Fuente: Elaboración de las autoras

Derivado de lo expuesto, es necesario puntualizar que cada habilidad fue observada por las investigadoras y permitió una valoración individual y grupal para que la docente pueda atender las particularidades de cada niño. Por ello, a la propuesta del trabajo colaborativo, presenta 5 de 75 con un nivel muy satisfactorio, los 25 se mantienen en un nivel satisfactorio y son 40 los casos que tienden a trabajar de forma aislada por el tipo de actividad que no propicia que se integren los pares para actuar; la meta es cumplir la tarea y el que primero termina tiene "legos" o rompecabezas como premio.

El segundo criterio a observar es la participación creativa, la misma que presentó 2 de los 75 niños con un resultado creativo, 30 tuvieron una producción que aportaron con algunos elementos novedosos y 43 restantes con apoyo cumplieron las consignas.

Los comportamientos interactivos, es otro de los criterios que fueron observados y 6 de los niños tuvieron una participación activa en todo el proceso de las tareas, 22 niños se observaron intermitencias y los 47 restantes se mantuvieron en sus puestos según la orden la maestra y cuando terminaban optaban por estar fuera del grupo.

Finalmente, el manejo de tolerancia entre los pares, presentó a 4 niños con un nivel muy satisfactorio, 38 de ellos obtuvieron un puntaje de poco satisfactorio. Estas prácticas dieron pauta para reflexionar en el sedentarismo, poca tolerancia, tendencia a aislarse en los 
trabajos, a frustrarse por no lograr los objetivos; no se motiva la curiosidad y los pares poco o nada se involucran en convivir con los demás.

\section{Resultados obtenidos de la encuesta aplicada a los docentes del nivel inicial}

Otro de los criterios que el estudio determinó necesario para la investigación, se realizó con una encuesta para valorar las prácticas de los docentes del nivel y los resultados se muestran en la figura 2.

Figura 2

\section{Manejo de las TIC según el nivel de frecuencia que tienen los docentes al momento de realizar sus acciones pedagógicas en la Escuela "Camino al Saber" de la ciudad de Guayaquil, 2018}

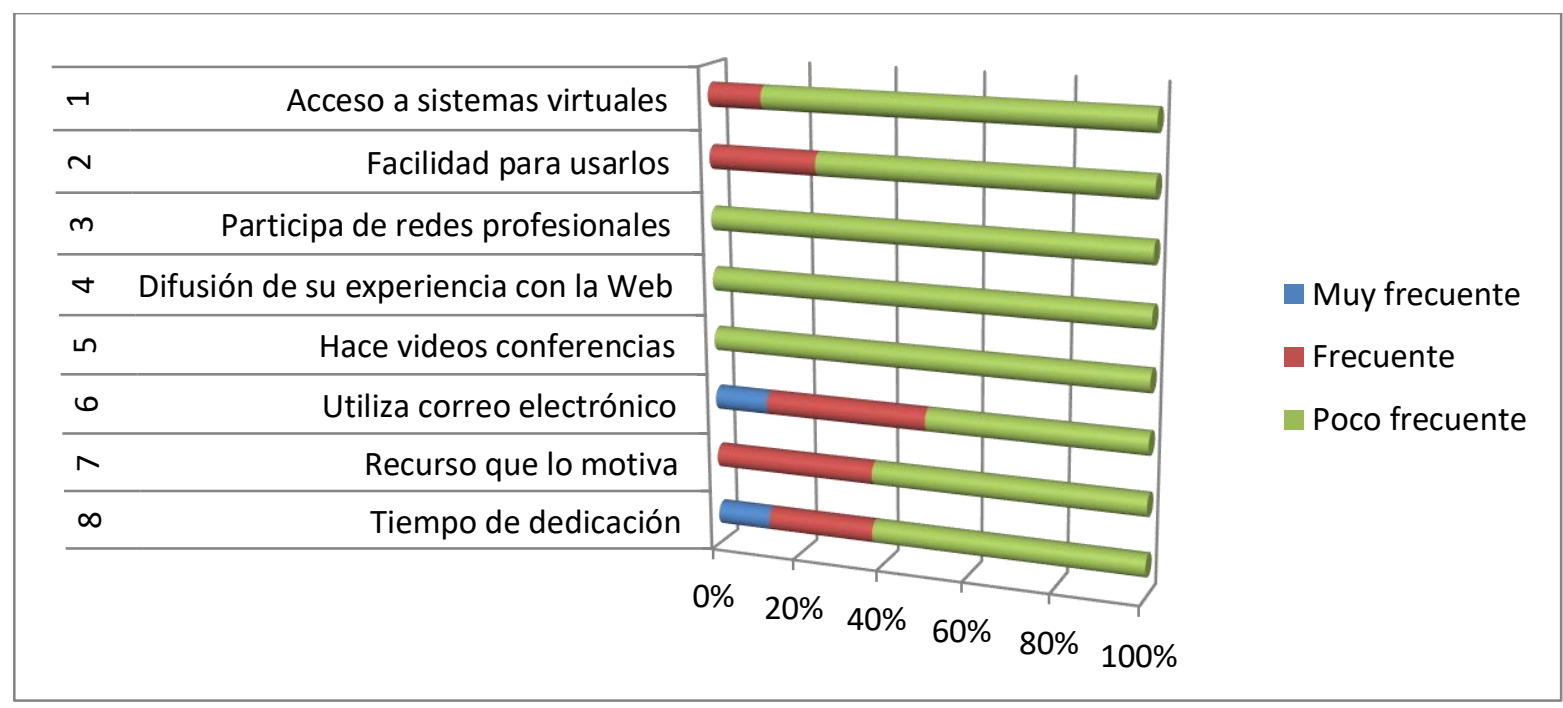

Fuente: Elaboración de las autoras

La figura 2 muestra resultados del nivel de conocimiento de los docentes en una de las competencias que no han sido consideradas en su gestión pedagógica y se registran los siguientes resultados:

En el criterio 1, Acceso a sistemas virtuales, respondieron el 12,5\% del 100\% con poco acceso a los sistemas virtuales.

En el criterio 2, Facilidad para usar los medios tecnológicos, se registró un $25 \%$ de respuesta que los docentes no tienen inconvenientes para usar las diferentes estrategias digitales. 
Del criterio 3, Participa de redes sociales, el 4 difusión sobre su experiencia en la web y el 5 hace video conferencias llamadas, reconocen los docentes que es escasa la intervención en redes sociales.

En el criterio 6, que corresponde al uso del correo electrónico, sólo el 12,5\% respondió que lo usa de forma muy frecuente, el resto en algún momento requirió crear su correo electrónico, pero lo usan de manera insuficiente.

En el criterio 7, un recurso que lo motiva, el $62,5 \%$ no se sienten fortalecidos para afrontar la competencia y por eso el 12,5 considera que les falta motivación en el uso de las TIC.

Todos estos resultados dan muestra de la urgencia que los docentes de educación inicial fortalezcan la competencia digital en su vida personal a fin que puedan hacerla parte de su práctica profesional.

\section{Resultados de la entrevista al directivo}

A partir de la información recabada en la entrevista, se reconoce que las TIC deben estar presente en los diferentes entornos educativos para que la convivencia áulica de los niños, tengan un aprendizaje significativo. Por todo ello, se direccionó para que el departamento de sistema junto con el DECE, programen capacitaciones a los diferentes miembros de la comunidad educativa, a fin que aprovechen al máximo la variedad de recursos que brindan las TIC y se sientan motivados por aprender, innovar y desarrollar las habilidades psicosociales en los entornos virtuales.

Por consiguiente, el carácter innovador de las TIC lleva a la docencia a estar alfabetizados en estos temas para que la falta de conocimiento en tecnología no sea un freno en la formación continua. Al hilo de lo señalado, Freire (1991) refiere que:

la educación no es reductible a la técnica, pero no se hace educación sin ella. No es posible, a mi punto de vista, empezar un nuevo siglo sin terminar este. Creo que el uso de ordenadores en el proceso de enseñanza aprendizaje, en cambio de reducir, puede expandir la capacidad crítica y creativa de nuestros niños y niñas. Depende de quién usa a favor de qué y de quién y para qué. (p.114)

De acuerdo al autor, se reafirma la necesidad de superar el atraso cultural y orientar los procesos. En este orden de ideas, las TIC son un recurso que mejora la comunicación, 
genera novedad, motivación, trabajo grupal; y en el direccionamiento óptimo del docente, propicia un ambiente de valores y aprendizaje permanente.

\section{Conclusiones}

A partir de las teorías consultadas y en consonancia con los hallazgos de la investigación, se determina el valor de las TIC en los procesos de enseñanza aprendizaje y la evidente influencia que tiene en el desarrollo psicosocial. Además, el participar en los escenarios virtuales en la etapa infantil, requiere que la comunidad educativa se capacite de forma continua para orientar la educación tecnológica de forma responsable y comprometida.

Por tal motivo, el uso de las TIC a temprana edad requiere que docentes y padres de familia tengan un control interno y externo para que los espacios de aprendizaje virtual cuenten con un camino efectivo.

Como reflexión final, se debe valorar que cada experiencia con las TIC, es un medio que se adapta a la naturaleza de cada niño y al interactuar con el entorno, les ofrece oportunidades para aprender y trascender.

\section{Referencias}

Alpízar, J. (2018). ¿De qué manera las nuevas tecnologías de la información (TICS) apoyan a la mejora de los aprendizajes en educación preescolar? Revista Atlante: Cuadernos de Educación y Desarrollo (febrero 2019). En línea. https://www.eumed.net/rev/atlante/2019/02/tic-educacion-preescolar.html

Briceño, L., Flores, R., \& Gómez, D. (2019). Uso de las TIC en Preescolar: Hacia la integración curricular. Revista Panorama, 13(24), 21-32.

doi: http://dx.doi.org/10.15765/pnrm.v13i24.1203

Bustamante, S. (2015). Currículo. Teoría y Diseño Curricular. Educación Inicial y Preparatoria. Quito-Ecuador: Primera edición. Obtenido de http://www.runayupay.org/publicaciones/curriculosbc.pdf

CERLALC. Centro Regional para el fomento del libro en América Latina y en el Caribe. Bajo los auspicios de la UNESCO. (2019). Lectura digital en la primera infancia. Obtenido de https://cerlalc.org/wp-content/uploads/2019/04/Dosier-Lectura-digital-VF3.pdf 
Fernández, A. (2016). Las nuevas tecnologías en la primera infancia. Obtenido de ( Trabajo fin de Máster en intervención psicológica en contextos de riesgo. Universidad de Cádiz):

https://rodin.uca.es/xmlui/bitstream/handle/10498/19823/FTM\%20Araceli\%20Fem \%C3\%A1ndez\%20Eslava.pdf?sequence $=1$

Freire, P. (1991). La Educación en la Ciudad. Sao Pablo, México: Siglo XXI, editores argentina,s.a. Obtenido de http://funama.org/data/PEDAGOGIA\%20CRITICA/freire/La\%20educacion\%20en \%201a\%20ciudad.pdf

Ledesma,Y., Cobos, A. (2018). La Triple Hélice de las TIC en Ecuador. Revista Ciencia e Investigación, 3(9), 41-48. https://revistas.utb.edu.ec/index.php/sr/article/view/257

Lemus, M. (2018). Programa de Psicoeducación para Mejorar el Desarrollo Social y Cognitivo en Niños. Obtenido de (Trabajo de titulación presentado como requisito para la obtención del título de Licenciada en Psicología. Universidad San Francisco de Quito.): http://repositorio.usfq.edu.ec/bitstream/23000/7165/1/137269.pdf

Roca, G. (Coord.). (2015). Las nuevas tecnologías en niños y adolescentes. Guía para educar saludablemente en una sociedad digital. Barcelona. Hospital Sant Joan de Déu (ed). Obtenido de https://www.adolescenciasema.org/wp-content/uploads/ 2015/06/cuaderno-faros-2015-es-TICs.pdf

UNESCO. (2013). Enfiques Estratégicos sobre las TICs en Educación en América Latina y el Caribe. Obtenido de TICs y Nuevas Prácticas Educativas: http://www.unesco.org/new/fileadmin/MULTIMEDIA/FIELD/Santiago/images/tics esp.pdf

UNICEF. (2017). Estado mundial de la infancia 2017. Niños en un mundo digital. Obtenido de: https://www.unicef.org/peru/spanish/Estado_Mundial_de_la_Infancia_2017._Ninos y ninas en un mundo digital. Resumen Ejecutivo - UNICEF.PDF 